\title{
Análisis de los elementos de mercadeo social implícitos en campañas de prevención de embarazo en adolescentes
}

\author{
Analysis of Social Marketing Implicit Elements in Adolescent \\ Pregnancy Prevention Campaign
}

\section{Análise dos elementos de marketing social implícitos nas campanhas de prevenção da gravidez na adolescência}

\author{
Ana María Restrepo-E. ${ }^{1}$, Yaromir Muñoz ${ }^{2}$, María Adelaida Duque-D. ${ }^{3}$ \\ 1 Diseñadora industrial, especialista en mercadeo y MBA. Universidad EAFIT. Colombia. arestr81@eafit.edu.co \\ 2 Psicólogo, especialista en Mercadeo, MBA y PhD en Administración. Universidad EAFIT. Colombia. ymunoz@eafit.edu.co \\ 3 Comunicadora social, especialista en Mercadeo y especialista en Estudios Políticos; magíster en Comportamiento \\ del consumidor. Universidad EAFIT. Colombia. mduqued1@eafit.edu.co
}

Recibido: 28/09/2015. Aprobado: 18/05/2018. Publicado: 30/05/2018

Restrepo-E, AM, Muñoz Y, Duque-D MA. Análisis de los elementos de mercadeo social implícitos en campañas de prevención de embarazo en adolescentes. Rev. Fac. Nac. Salud Pública. 2018;36(2):18-27. Dor:10.17533/udea.rfnsp.v36n2a04

\section{Resumen}

Objetivo: Este artículo analiza dos campañas orientadas a prevenir el embarazo en adolescentes, con el fin de elucidar la incorporación de elementos procedentes de disciplinas como el mercadeo, y cómo estos pudieron incidir en los logros de las mismas, a la luz del modelo de "la rueda de mercadeo social". Metodología: Se revisaron informes de estudios cualitativos y cuantitativos sobre las campañas "Sexo a lo Bien” y "Parents Speak Up!", realizadas en dos contextos sociales diferentes: una en Colombia y la otra en Estados Unidos. El procedimiento de análisis fue el rastreo de elementos de mercadeo implícitos en ambas campañas, a partir de los archivos identificados sobre las mismas. Resultados:
Se evidencia que la falta de coherencia entre la definición del problema, la estrategia usada y la ejecución de la misma afecta los resultados esperados en una de las campañas. La campaña "Parents Speak Up!" presenta logros importantes, porque se basó en un buen respaldo teórico, articulado con una ejecución bien definida frente a distintos públicos focalizados. La menor efectividad de resultados en la campaña "Sexo a lo Bien" se debe a la ambiciosa propuesta, así como a la falta de coherencia entre la concepción del problema que se enfrenta y la poca claridad en la definición de públicos de interés y en el contenido de los mensajes. Conclusiones: Para el logro efectivo de resultados en campañas educativas 
sobre prevención de embarazo en adolescentes, el mercadeo social puede aportar elementos en cuanto a incorporar el proceso de planeación, ejecución y evaluación respecto del tipo de producto, el público y el contenido de los mensajes.
-Palabras clave: embarazo en adolescencia, mercadeo social, programa de educación sexual, comunicación en salud, comportamiento sexual.

\section{Abstract}

Objective: this article analyses two campaigns designed to prevent adolescent pregnancy. The authors aim to clarify the implementation of elements coming from other disciplines such as marketing, and the possible effects in the campaigns' results, in light of "the social marketing wheel" model. Methodology: reports of qualitative and quantitative studies on the campaigns "Sexo a lo Bien" and "Parents Speak Up!" were studied. Those campaigns were originally implemented in different contexts: one in Colombia and one in the United States. The analysis was carried out by tracking implicit marketing elements in both campaigns, from existing records about them. Results: a lack of coherence between the problems' definition, the chosen strategy and its execution was evident and affected the expected outcomes in one of the campaigns. The "Parents Speak Up!" campaign achieved important results, because it had a strong theoretical basis, articulated with a well-defined execution for different target focused audiences. The "Sexo a lo Bien" campaign had less effective results. It was too ambitious; it lacked coherence between the aimed problem, an unclear definition of target audience and the content of the messages. Conclusions: Social marketing can offer valuable elements for and educational campaign on adolescent pregnancy to achieve effective results, such as planning, execution and evaluation of the type of product, the target audience and the content of the message.

Key words: adolescents' pregnancy, social marketing, sexual education program, health communication, sexual behavior

\section{Resumo}

Objetivo: este artigo analisa duas campanhas encaminhadas a prevenir a gravidez na adolescência, visando esclarecer a incorporação de elementos originários de disciplinas como o marketing, e seus possíveis efeitos nos resultados das campanhas, utilizando o modelo de "a roda de marketing social". Metodologia: revisaram-se relatórios de estudos qualitativos e quantitativos sobre as campanhas "Sexo a lo Bien" e "Parents Speak Up!", realizadas em dois contextos sociais diferentes: uma foi feita na Colômbia e a outra nos Estados Unidos. A análise foi mediante rastreio de elementos de marketing implícitos nas duas campanhas, com arquivos identificados sobre elas. Resultados: a falta de coerência entre a definição do problema, a estratégia utilizada e a sua execução foi evidente, afetando os resultados esperados em uma das campanhas. A campanha "Parents Speak Up!" obteve resultados importantes, porque teve uma forte base teórica, articulada com uma execução bem definida para diferentes públicos focalizados. A campanha "Sexo a lo Bien" teve resultados menos efetivos por ser uma proposta muito ambiciosa, e por falta de coerência entre a concepção do problema objetivo, uma definição difusa de públicos de interesse e o conteúdo das mensagens. Conclusões: para conseguir resultados efetivos nas campanhas educativas sobre a prevenção da gravidez na adolescência o marketing social pode fornecer elementos relacionado com o processo de planejamento, execução e avaliação do tipo de produto, do público alvo e do conteúdo das mensagens.

---------Palavras-chave: gravidez na adolescência, marketing social, programa de educação sexual, comunicação em saúde, comportamento sexual.

\section{Introducción}

El embarazo precoz en adolescentes es una preocupación a nivel mundial, sobre todo para las naciones en desarrollo. Los registros de la Organización Mundial de la Salud (OMs) reportan que cerca de 16 millones de adolescentes (entre 15-19 años) y 1 millón de preadolescentes (menores de 15 años) dan a luz anualmente, de manera primordial en países con ingresos medios o bajos [1]. En Colombia se registra que, en 2010, el $19,5 \%$ de las adolescentes de edades entre 15 y 19 años ya eran madres o se encontraban en embarazo, y para el 2015, ese porcentaje descendió a 17,4 \%. Para el caso de la ciudad de Medellín, la tasa se ubica en 12,8 \% [2]. Estos indicadores han llevado a emprender actividades preventivas, mediante esfuerzos institucionales, en el marco de un proyecto social que integra diversos niveles de atención [3]. Dichas intervenciones masivas tienen 
un alto componente de tipo persuasivo motivacional e informativo para prevenir el embarazo en adolescentes.

Este estudio se enfoca en analizar dos campañas de manejo preventivo del embarazo en adolescentes, una fue realizada en Colombia y la otra en Estados Unidos, con el fin de elucidar la incorporación de elementos procedentes de disciplinas como el mercadeo, y cómo estos pudieron incidir en los logros de las mismas, a la luz del modelo de "la rueda de mercadeo social" [4], en las fases de planeación, desarrollo y evaluación de dichas intervenciones.

El mercadeo social es una derivación del uso de conceptos de mercadeo comercial, con fines diversos, como lograr cambios en conductas de los individuos, las cuales, por su naturaleza y arraigo, pueden resultar perjudiciales para el bienestar individual y social [5]. La forma de operar es con intervenciones masivas, en busca de promover cambios de actitud y de comportamiento en los públicos focalizados.

El mercadeo combina varios aspectos, como el producto, la comunicación, la plaza y el precio; en este caso, el producto es una idea centrada en un mecanismo de autoprotección que apela al autocontrol individual. Dicha idea se promueve con un plan de comunicación y de acciones orientadas a una población definida, en un contexto territorial determinado y a un costo implícito [6], que puede ser entendido como capacidad de controlar, evaluar y asumir consecuencias.

En Medellín, varias instituciones, como la Alcaldía y la Red de Prevención del Embarazo Adolescente, y organismos multilaterales, como el Banco Interamericano de Desarrollo y la Agencia de Cooperación Española, preocupadas por el incremento de embarazos en jóvenes, se unieron para desarrollar, en 2007, la campaña "Sexo a lo Bien", cuya finalidad fue trabajar en la sensibilización y la movilización social respecto a temas como el embarazo precoz y la sexualidad adolescente, partiendo de una concepción holística y sobre una línea de base de la magnitud del problema que orientara los esfuerzos [7]. El manejo comunicacional de la campaña se hizo en medios masivos y, de modo más interactivo, a través de su página web. El público focalizado fue de adolescentes, padres de familia y red escolar, que se compone de los veinticuatro núcleos educativos, uno por cada comuna de la ciudad. La campaña se enmarca en el proyecto "Sol y Luna", cuyo enfoque es la prevención con un doble propósito: contribuir a la disminución de la problemática de embarazo adolescente y mejorar el conocimiento de los jóvenes sobre salud sexual y reproductiva.

En Estados Unidos también existe la preocupación por el problema del embarazo adolescente. Por ello, se indagó por conocer la opinión de diferentes estamentos sobre las implicaciones del ejercicio de la sexualidad en la adolescencia, y se encontró que el $94 \%$ de los adultos y el $92 \%$ de los adolescentes estaban de acuer- do con que era importante que estos últimos reciban un mensaje de la sociedad sobre que no deben tener relaciones sexuales hasta que al menos hayan terminado la escuela secundaria [6]. Este estudio fue el detonante para que, en junio de 2007, se lanzara a nivel nacional la campaña "Parents Speak Up!", la cual involucró una comunicación multimedios, con el objeto de incrementar el número de padres que hablan con sus hijos preadolescentes acerca de retrasar, con fines preventivos, el inicio de las relaciones sexuales, ya que esto se ha identificado como uno de los factores que ayudaría en la disminución de la problemática.

Ambas campañas buscaron incidir positivamente en disminuir la problemática del embarazo en adolescentes, pero cada una contó con su propio direccionamiento respecto de su fin. En este sentido, esta investigación pretende responder a la siguiente pregunta: ¿cuáles son los aspectos de mercadeo social de las campañas analizadas, que contribuyen a lograr efectos positivos en la disminución de la problemática?

El embarazo precoz en adolescentes se aborda, con fines académicos y de intervención, desde aspectos biológicos, como la edad temprana de la menarquia, ligados a la falta de una adecuada educación sexual y cuidado de los padres, especialmente en fases tempranas; adolescentes con perspectivas económicas muy limitadas; la incapacidad de rehusarse a tener relaciones sexuales no deseadas, o tenerlas bajo efectos del alcohol [7]; la baja propensión al uso de medios anticonceptivos, a pesar de tener acceso a ellos [8]. Igualmente, se identifican, como causas que posibilitan el embarazo adolescente, factores como el inicio precoz de las relaciones sexuales; los encuentros intergeneracionales; las ideas en torno al amor, así como los tipos de relación de pareja [9], y variables de tipo cultural, entre los cuales se evidencia la influencia del entorno con familias nucleares desestructuradas, además de ambientes escolares donde la presión de los compañeros sobre el comportamiento íntimo es alta [10].

\section{El rol del colegio y de las familias en el comporta- miento sexual activo de adolescentes}

Prevenir en salud sexual involucra reconocer el rol formativo de las escuelas y la importancia de los proyectos de vida como opciones para reducir el embarazo adolescente y promover, en los jóvenes, la salud sexual [11]. Esto, sumado a sanas relaciones con los padres y otros adultos en cuanto a comunicaciones fluidas, se asocia con una disminución de la conducta sexual riesgosa [12] y que puede lograr que el comportamiento sexual activo de los adolescentes se retrase en su iniciación [13]. Sin embargo, se señala que toda esta influencia se da es en los preadolescentes, y que entre los adolescentes se evidencia que tanto los pares como los medios de comunicación tienen mayor impacto en su comportamiento [14]. No obstante, 
se ha identificado que la supervisión de los padres es esencial para una experiencia responsable de la sexualidad en la adolescencia $[15,16]$.

En cuanto a la efectividad de los programas de educación centrados en la abstinencia, así como entre los que promocionan el uso de medios anticonceptivos, existe un debate continuo. Algunos estudios sugieren que estos son ineficaces y pueden promover actitudes negativas hacia el uso del condón y a otros comportamientos de prevención de riesgos [17], mientras que otros han encontrado que dichas intervenciones inciden en menores tasas de actividad sexual $[18,19]$, y que traen consigo ventajas personales, sociales y educativas $[20,21]$ en cuanto al modo de comportarse en lo relacionado al ejercicio de la sexualidad.

\section{Intervenciones con apoyo de mercadeo social}

Para aportar a prevenir la problemática del embarazo adolescente existen programas e intervenciones en niveles primarios con el uso del mercadeo social, a nivel educativo, centrado en escuelas y colegios, y también intervenciones a núcleos familiares [22]. Las evidencias de casos estudiados muestran que campañas de este orden funcionan para fomentar la emulación de comportamientos y hábitos saludables [10,11].

En el mercadeo orientado a temas de salud se utilizan mensajes persuasivos, fundamentados en una clara segmentación de públicos que guíe la comunicación, la cual se hace en función de factores conductuales, demográficos y psicográficos que se identifican en la base del comportamiento nocivo y el deseado $[5,6]$. Esto se encuentra en la propuesta de la "rueda de mercadeo social" [4], esquema teórico propuesto para el análisis de las campañas en este trabajo, el cual plantea una disciplinada aplicación de seis pasos: planificación y desarrollo de la estrategia, selección de canales y materiales, pruebas preliminares, aplicación del programa, evaluación de la eficacia y perfeccionamiento de los materiales para el futuro, los cuales deben seguirse rigurosamente, para augurar resultados positivos en cuanto a cambios de comportamiento. Estas intervenciones sociales mediáticas se planifican usando como base la teoría del comportamiento; luego se define el contenido del mensaje y el medio apropiado, lo cual exige conocer bien la audiencia a la que se dirige y el tipo de comportamiento a cambiar, así como el grado de implicación de los individuos.

La aplicación de mercadeo social para cambiar comportamientos requiere continuidad y claridad en los objetivos a lograr [4,5], con un amplio horizonte temporal y un sistemático esfuerzo en las variables de producto, comunicación, plaza y costo que representa para los individuos, que en aspectos sociales puede ser el sacrificio de no hacer algo o la postergación de comportamientos.

\section{Metodología}

El procedimiento empleado es un análisis descriptivo de dos campañas, llevado a cabo con el fin de, mediante la revisión de reportes de caso, identificar los aspectos de mercadeo implícitos en las mismas, que aportan a propiciar cambios en cuanto al ejercicio de una sexualidad responsable en adolescentes, y los elementos que permiten el logro de los objetivos en cuanto a cambios de conducta. Se seleccionaron, para el estudio, dos campañas: "Sexo a lo Bien", llevada a cabo en Medellín, y "Parents Speak Up!", realizada en Estados Unidos.

En cuanto al alcance de la revisión de reportes de caso, se deja explícito que se analizan las campañas desde la incorporación que hagan de los aspectos de mercadeo, sobre la base de reconocer que se presentan en contextos disímiles y en ámbitos culturales y sociales diferentes.

El análisis recurrió, como fuentes, a reportes evaluativos y publicaciones de tipo científico sobre las campañas, que facilitaran información acerca del desarrollo, la implementación e impactos de cada una de ellas desde la perspectiva de quienes ejecutaron el trabajo, y que permitieran la discusión y el análisis bajo una mirada desde el marco teórico de mercadeo social [4]. Este análisis consta esencialmente de tres momentos: en un primer momento se identifica la planeación de las campañas, donde se incluye la definición y la fundamentación conceptual, declaración de objetivos y de estrategia. Un segundo momento se ocupa del desarrollo, en el cual se incluyen aspectos como definición de público al que se dirige y los medios usados, así como el contenido de mensajes ejecutados. El tercer momento implicó la evaluación de los mismos proponentes de las campañas, con el propósito de analizar los logros alcanzados en cuanto a lo planeado.

Sobre la campaña "Sexo a lo Bien" se encontraron estudios publicados en revistas de divulgación científica [3], de los cuales algunos se ocuparon de establecer una línea de base con diseño experimental para determinar la magnitud del problema [7,9]. Se tomó también como fuente un estudio que sistematiza, de manera muy global, el proyecto en el que la campaña se inscribió [23], así como un estudio efectuado por los promotores para evaluar resultados cuantitativos de recordación y efectividad de los medios empleados [24], y un reporte acerca del componente comunicativo de la campaña [25].

Para la campaña "Parents Speak Up!" se tuvieron en cuenta cuatro fuentes secundarias disponibles, una de ellas sobre los resultados del estudio experimental que se hizo previo al lanzamiento de la campaña [26] y que midió, de forma cuantitativa, y en un ambiente controlado, las reacciones y los cambios asociados a ella; el segundo 
estudio reporta, también de manera cuantitativa, la efectividad real de la campaña [27]. Adicionalmente, se revisó un estudio posterior sobre los efectos de la campaña en la conversación entre padres e hijos [28] y se tuvo en cuenta el análisis de estrategia, con enfoque de mercadeo social, publicado por otros investigadores [6].

Por la naturaleza pública de las fuentes consultadas de tipo secundario y el tipo de análisis efectuado, este estudio no requirió aprobación por parte del Comité de ética de la Universidad EAFIT.

\section{Resultados}

\section{Campaña "Sexo a lo Bien"}

Esta campaña fue desarrollada en Medellín, Colombia, en el marco del proyecto "Sol y Luna", en un lapso de 24 meses. La campaña se centró en la atención preventiva del embarazo en adolescentes.

En la tabla 1 se presenta el análisis de la campaña "Sexo a lo Bien" desde la perspectiva de mercadeo social.

Tabla 1. Análisis de la campaña "Sexo a lo Bien" desde la perspectiva de mercadeo social.

\begin{tabular}{|c|c|}
\hline \multirow{3}{*}{ Planeación de la campaña } & $\begin{array}{l}\text { Fundamentación teórica: no hay trazabilidad de marco teórico conceptual de respaldo a los } \\
\text { contenidos de la campaña. } \\
\text { Se plantea una visión holística de la campaña con tres componentes [28]. } \\
\text { Se realizó trabajo de campo previo para identificar aspectos como mitos, miedos y formas } \\
\text { de vivir la sexualidad en los jóvenes; se estableció una línea de base sobre el tamaño del } \\
\text { fenómeno [7,9]. } \\
\text { Se evidencia que la campaña responde a atender el fenómeno como conjunto de hechos y } \\
\text { representaciones sociales. } \\
\text { Definición del problema: se parte de la percepción que tienen padres e hijos sobre el } \\
\text { embarazo adolescente, que no garantiza relaciones estables y trunca proyectos de vida. }\end{array}$ \\
\hline & $\begin{array}{l}\text { Objetivos: el enfoque fue sensibilizar, desmitificar y legitimar la sexualidad en adolescentes. } \\
\text { También buscaron informar y motivar sobre la importancia del uso de métodos } \\
\text { anticonceptivos y apoyar aspectos de equidad de género. }\end{array}$ \\
\hline & $\begin{array}{l}\text { Estrategia: hubo tres componentes en la campaña [28]: } \\
\text { 1. Mejorar la oferta de servicios de salud sexual y reproductiva para jóvenes. } \\
\text { 2. Comunicación y movilización social. } \\
\text { 3. Investigación, evaluación y difusión de resultados. } \\
\text { El componente } 2 \text { es el que interesa desde mercadeo social. El enfoque propuesto apuntó a } \\
\text { tres aspectos: a) denunciar, mediante el uso de cifras altas de embarazo adolescente; } \\
\text { b) evidenciar el problema, recurriendo al uso de imágenes de adolescentes en embarazo, } \\
\text { y c) realizar cuestionamientos sobre las responsabilidades que implica estar embarazada; } \\
\text { luego se pasó al proceso de confrontar e institucionalizar [24], donde se interrogaba qué se } \\
\text { estaba haciendo al respecto para enfrentarlo. } \\
\text { La campaña declara que se priorizó el tema de salud más que el de educación. }\end{array}$ \\
\hline \multirow[t]{2}{*}{ Desarrollo } & $\begin{array}{l}\text { Se implementó la estrategia con comunicaciones en medios masivos, en forma fiel a los } \\
\text { diversos objetivos y a públicos de todo tipo. } \\
\text { Segmentación: un público heterogéneo, amplio, sin que fuese definido algún criterio de } \\
\text { homogeneidad de la población. }\end{array}$ \\
\hline & $\begin{array}{l}\text { Canales: utilizó múltiples canales, como medios de comunicación nuevos y tradicionales. } \\
\text { Usó página web y línea telefónica. } \\
\text { El lenguaje y los contenidos se definieron en función de la gente joven. }\end{array}$ \\
\hline \multirow[b]{2}{*}{ Evaluación } & $\begin{array}{l}\text { Sobre los objetivos: se planteó el incremento en el uso de métodos anticonceptivos y la } \\
\text { disminución del embarazo adolescente. }\end{array}$ \\
\hline & $\begin{array}{l}\text { No se definieron criterios sobre el nivel de exposición a los medios ni la frecuencia. } \\
\text { No se definieron elementos para saber si el mensaje era comprendido. } \\
\text { Sí se declaró medición de resultados de la intervención a partir de la línea de base y } \\
\text { posterior medición tras la campaña. Se previeron cambios en conocimientos y en actitudes. }\end{array}$ \\
\hline
\end{tabular}

Adicional a lo que se analiza en la tabla 1, se resalta que, en cuanto a recordación espontánea sobre el contenido de la campaña, el lenguaje usado durante la ejecución no logró recordación inmediata en ninguno de los públicos estudiados (jóvenes, expertos, formadores de opinión, padres de familia y ejecutoras), y que más del $50 \%$ de las usuarias no recordaban ni el logo ni las imágenes asociadas a ella, a pesar de su participación [24]. 
Los expertos, participantes en el reporte mencionado, manifestaron reserva frente al tema de asociar sexualidad con genitalidad por medio de una imagen que explicitaba, mediante la caricatura, los órganos reproductivos de ambos sexos; adicionalmente, algunos de ellos consideraron que la imagen de la campaña no tenía nada que ver con el contenido, dada la complejidad del tema, o que era muy ambigua en su mensaje. Frente a esto, hay posiciones diferentes: por un lado, la mayoría de los formadores de opinión coincidieron con que la imagen transmitía mensajes negativos, por las asociaciones que generaba y, por otro lado, el 82,28 \% de los jóvenes evaluó la imagen como totalmente adecuada [24].

El lenguaje de la campaña, por su parte, es calificado por las ejecutoras como apropiado para los jóvenes y de choque para adultos, al considerarlo como promotor del sexo; mientras que, para los expertos, es fuerte, llama a la reflexión y genera controversia con temas éticos. Según afirmaciones de agentes de influencia, como padres de familia y educadores, la campaña promovía, mediante su lenguaje, comportamientos opuestos a lo que asumen como sano y adecuado [24]. Se encontró que una de las principales falencias de la comunicación fue que la estrategia de intervención se enfocó en el sector salud, cuando el problema es de educación, y que el proceso comunicativo fue funcionalista, de carácter informativo más que formativo [25].

La participación de los actores para plantear sus necesidades en materia de educación sexual fue baja; allí se visibilizan particularmente elementos de falta de claridad en la definición del problema.
En cuanto a la efectividad publicitaria, se observa que las usuarias no manifestaron impactos respecto a un mayor conocimiento o acceso a nueva información, así como tampoco se generaron corrientes de opinión [24], y a pesar de que se reportan resultados positivos en lo que tiene que ver con las variaciones en las creencias previas, aspectos metodológicos como la pérdida de un buen porcentaje del grupo de encuestados de la línea de base y, por lo tanto, su reemplazo, no permiten que estas puedan ser atribuidas a la campaña "Sexo a lo Bien" [24].

Por otra parte, la campaña, en medios masivos, fue sensibilizadora, pero no hay evidencias de cambios en los comportamientos o en las actitudes atribuibles a la campaña respecto a la edad de inicio de las relaciones sexuales, del uso de los métodos anticonceptivos, vivencia de las relaciones de poder y disminución de la presencia de los estereotipos de género, a lo que se le sumó un incremento en el reporte de enfermedades de transmisión sexual [23].

\section{Campaña "Parents Speak Up!"}

Esta campaña tiene su inicio con los hallazgos acerca de la abstinencia y de la incidencia que tiene sobre el tema la comunicación entre padres e hijos, y desarrolló un argumento orientado a postergar la iniciación de la actividad sexual, mediante la comunicación de las ventajas de la espera y los riesgos para la salud de la iniciación sexual temprana.

En la tabla 2 se presenta el análisis de la campaña “Parents Speak Up!” desde la perspectiva de mercadeo social.

Tabla 2. Análisis de la campaña "Parents Speak Up!" desde una perspectiva de mercadeo social.

Fundamentación teórica: la teoría de cambio de comportamiento asociada a la escuela cognitiva de psicología es la que sostiene la propuesta de trabajo; allí se da mucho valor a las normas subjetivas [6]. Se hace referencia al índice de la norma social de esperar una edad más adulta para las relaciones sexuales.

Se tuvieron en cuenta estudios previos sobre las prácticas comunicativas entre padres e hijos.

Estudios previos indican que el tema de inicio de relaciones sexuales era poco abordado en la comunicación.

Definición del problema: la sociedad estadounidense consideraba problemático el inicio precoz de las relaciones sexuales en adolescentes.

Planeación de la campaña

Objetivos: alcanzar, después de un año, un incremento del 20 \% en el número de padres que hablen con sus hijos preadolescentes acerca del momento indicado para tener relaciones sexuales.

Estrategia: se puso a prueba el contenido de los mensajes de la campaña y se validó la efectividad de estos antes de implementarse.

Contenido: sensibilizar, motivar a abordar el tema en familia y facilitar que se hable de él, evidenciar beneficios de la abstinencia y del retraso en el inicio de la vida sexual con pareja, centrarse en la comunicación entre padres e hijos, y usar medios masivos para extender el mensaje. 


\begin{tabular}{ll} 
Continuación Tabla 2 & \\
\hline Primer componente, uso de medios: los canales centrales fueron la televisión y la \\
radio (anuncios de servicio público), apoyados por publicidad exterior, publicidad en \\
autobuses, carteles, banners web (formato de comunicación digital que se usa en sitios \\
web), y kits de medios, teniendo en cuenta los hallazgos acerca de la influencia de \\
campañas mediáticas para movilizar conductas en los padres. \\
Como segundo componente se creó un sitio web para proporcionar, paso a paso y a \\
través de guías, información a los padres sobre cómo hablar con sus hijos acerca de \\
esperar para tener relaciones sexuales y de cómo ayudarlos en este proceso, además \\
de otros temas relacionados como los proyectos de vida, la realización profesional, las \\
relaciones sanas, entre otros. \\
Público objetivo: se tuvieron en cuenta variables como edad, género y raza. El material \\
de la campaña fue diseñado para el público objetivo, padres con hijos preadolescentes \\
de entre 10 y 14 años, y adaptado de acuerdo con públicos procedentes de grupos \\
subculturales, como los afroamericanos, indoamericanos e hispanos [26]. \\
Se definió evaluar la conciencia generada gracias a los impactos inmediatos de la \\
comunicación entre padres e hijos [27] y también el índice de expectativas de largo \\
plazo. \\
Se definió evaluar el impacto de los mensajes en aspectos conductuales que obedecen \\
a dicha campaña y también evaluar los efectos del mensaje en las interacciones \\
conversacionales padres e hijos [27,28]. \\
Se definió en porcentajes, y en un tiempo definido, el logro esperado acerca de que \\
padres e hijos hablaran del tema.
\end{tabular}

Los hallazgos sugieren que las interacciones conversacionales con los padres (el producto que se definió en el manejo de campaña), y esperar a ser mayor de edad, ligado a las expectativas de resultados a largo plazo, fueron los dos factores cognitivos más afectados por los mensajes de la campaña, y que, además, fueron un fuerte predictor de la comunicación efectiva entre padres e hijos [27]. Puede evidenciarse que la campaña logró impactar la parte cognitiva comportamental de la población objetivo [28]. Así mismo, se encontraron diferencias significativas en los efectos de la exposición a los mensajes entre las madres y los padres, lo cual se explica por diferencias de género en el procesamiento de los mensajes y como respuesta a la orientación femenina que tuvo la campaña, soportada en hallazgos de que las madres están significativamente más involucradas en la educación sexual de sus hijos en comparación con los padres [26].

A pesar de que inicialmente los anuncios televisivos se restringieron a franjas publicitarias donadas por las cadenas de televisión local, lo que limitaba la exposición, se encontró que el 59,4\% de los padres informó haber visto u oído al menos uno de los anuncios y que esta conciencia de la campaña parecía estar asociada con los puntos brutos de índice de audiencia (rating), lo que sugiere una alta recordación.

Con base en registros de índice de audiencia, se estima que cada persona recibió, en promedio, veintidós exposiciones totales de anuncios o al menos una exposición por mes, y aunque se trata de un modesto nivel, los resultados sugieren que fueron suficientes para lograr algunos efectos positivos medibles en la comunicación entre padres e hijos, y muestran una correlación significativa entre ello y la campaña [27]. La escala de comu- nicación midió el inicio de conversaciones acerca de la actividad sexual, las charlas sobre esperar hasta estar casado para ser sexualmente activo y si se ha recomendado al hijo esperar para tener sexo. Se evidencia también que la conciencia acerca de la frecuencia de exposición a los anuncios televisivos estuvo fuertemente asociada con los niveles de comunicación entre padres e hijos y que el incremento de la frecuencia de exposición generaba mejores niveles de comunicación, sugiriendo una relación de "dosis-respuesta" entre los mensajes de la campaña televisiva y la conducta de los padres. Esta relación se encontró tanto para padres como para madres, y respecto a los anuncios televisivos, no siendo así para los anuncios en la radio, con los que no se encontraron asociaciones significativas [27].

\section{Discusión}

Afirmar que un programa de intervención social para cambiar conductas tiene éxito exige demostrar los efectos en la conducta que se quiere cambiar y que el resultado puede ser replicado [29]. Ello lo convierte en una buena práctica que, desde el punto de vista de mercadeo social, puede considerarse un aprendizaje de capacidades en colectivo. Un primer momento clave en el desarrollo de programas de intervención social es identificar las variables que funcionan para resolver la situación problemática [14,28,29]. Segundo, es fundamental tener en cuenta que los logros obedecen no solo a esfuerzos institucionales, sino que es capital el papel de la familia en una conversación directa y que confronte a la vez que enseña [8,10,27], así como la creación de capacidades en los actores comprometidos, 
en una red armonizada bajo un liderazgo coherente en acciones que apuntan a brindar el "producto y la comunicación" a sus públicos [30] desde lo que se define en la estrategia de trabajo.

Es importante que las intervenciones se realicen desde edades tempranas, por sus efectos a largo plazo, y bajo la premisa de que la efectividad de los mensajes de prevención es menor en conductas ya instaladas en los individuos [31] y mayor si el comportamiento no se ha presentado (esto es, los estudios sobre prevención del tabaquismo, por ejemplo, suelen ser muy eficaces en quienes aún no fuman).

Se ha encontrado que los programas de éxito en promoción y prevención para adolescentes se concentran en una o dos conductas, se basan en un modelo teórico de apoyo, tienen continuidad en el tiempo y la información que entregan es verdadera y personalizada, de la misma manera que es esencial el hecho de que el público se sienta implicado y sea puesto en situación $[22,32]$, de tal forma que permita aprehender el problema y participar de la solución.

Si bien estos aspectos son relevantes, también lo es que el público que recibe la formación basada en información tenga habilidades cognitivas para incorporar lo que le proponen, y frente a esto, los adolescentes no tienen, necesariamente, la historia y la madurez cognitiva para procesar en profundidad lo que reciben. Algunos aspectos señalados, en otros estudios, muestran que las dificultades existentes en lograr resultados dependen de las dificultades individuales para internalizar cognitiva y comportamentalmente la práctica de la promoción en salud [33], ligado esto a la desconfianza que sienten ante las capacidades y habilidades del personal que brinda atención primaria, porque, en algunos casos, aparecen juicios de valor sobre su conducta sexual.

Se pueden evidenciar, mediante este trabajo, lecciones aprendidas desde el punto de vista de mercadeo social, provenientes de ambas campañas. La secuencia del proceso, preconizada por la disciplina del mercadeo, se hace muy evidente en la campaña realizada en Estados Unidos, donde además se evidencian publicaciones académicas que reflexionan sobre lo ocurrido [6].

En el caso de la campaña "Sexo a lo Bien", se pueden resaltar aspectos del alcance, la evaluación y los resultados, y sobre todo que responde a un plan mayor de trabajo, con la mirada puesta en el largo plazo y con un enfoque holístico [7]; de allí sus resultados en registros de menores tasas de prevalencia respecto de la línea de base fijada e incremento en el uso de métodos anticonceptivos, al igual que el flujo de adolescentes que buscaron apoyo en centros de atención [32]. A pesar de que la infraestructura destinada para esto no es muy conocida por algunos, les da pereza acudir a ellos [7] o sienten desconfianza porque perciben que el personal de salud no está capacitado para atenderlos y suelen juzgarlos [33]. Existe otra perspectiva a considerar: el hecho de brindar solamente la información sobre los riesgos y las consecuencias no es suficiente [34], por lo que se requiere estar muy activos con el apoyo escolar y familiar a los adolescentes.

Desde nuestra perspectiva, la campaña realizada en Medellín, en su componente comunicacional, no logra ajustarse a lo que, desde el mercadeo social, debiera organizarse metodológicamente, dada la amplitud y la ambición de objetivos que plantea su visión holística. Su naturaleza, como aparece definida, da opciones de un programa de intervenciones de más largo plazo y se esperaría, por tanto, mayor amplitud y adecuada preparación del personal en las otras fases que son más centradas en atención primaria. Aunque el público meta de la campaña fue la ciudadanía en general [23], específicamente de las comunas y barrios focalizados, los encuestados participantes en la evaluación de la campaña tuvieron distintas consideraciones frente al segmento que creían al que la campaña se dirigía, pues un número importante de respuestas señalaban que las mujeres eran las destinatarias exclusivas de los mensajes, mientras que un $44,5 \%$ pensaba que estaba dirigida a adolescentes y jóvenes [24]. La evidencia señala que la participación en la campaña fue baja en alcance [23], en parte debido a la forma comunicacional funcionalista, que no facilitaba interactividad [25]. Todo ello puede ser atribuido a que el público objetivo definido para la campaña fue muy amplio, con unas necesidades y características tan heterogéneas que no logró que todos se sintieran representados en ella [24].

En una época donde la existencia en mundo digital es casi tan importante como la existencia real, el uso de la página web genera envolvimiento, ya que, como lo muestra la campaña realizada en Estados Unidos, no solo incluía información y herramientas que ponían a los padres en situación, como se sugiere, sino que las guías creadas para orientar el trabajo se diseñaron de manera distinta según las características culturales de los segmentos, logrando la personalización que también se advierte importante. Estas guías, además, abordaban temas con incidencia positiva en la conducta sexual de los adolescentes, como las aspiraciones, las expectativas, la formación profesional, la preparación para la vida y sus perspectivas futuras.

\section{Conclusiones}

Partiendo de la evaluación de eficacia, es posible definir que la campaña: "Parents Speak Up!" logró efectos visibles en aspectos que inciden en el comportamiento sexual de los adolescentes, lo que a su vez se traduce en éxito, si se tiene en cuenta que dicho comportamiento 
está relacionado con la influencia de la campaña sobre la conducta que se quiere impactar, no siendo así para "Sexo a lo Bien". Ambas campañas se valieron, de manera implícita, del mercadeo social en función de la sexualidad responsable en adolescentes; sin embargo, al obtenerse de ellas resultados tan distintos a pesar de una "misma" intención, es importante detenerse en la forma como se llevaron a cabo, desde su concepción hasta su implementación, para entender en dónde radicaron y cuáles fueron las diferencias que marcan los logros de una y la baja efectividad de otra.

La campaña "Parents Speak Up!'” se sirvió de una teoría de comportamiento que garantizara una orientación adecuada; definió su problema a partir de la comunidad que deseaba impactar, y apoyó dicha definición desde la evidencia científica. Además, se concentró en un objetivo específico, claro y medible en un tiempo determinado; su segmentación fue pertinente, permitiendo adaptar la campaña a otros subgrupos cuando se requería; sus canales y materiales obedecieron a los objetivos y la segmentación; pruebas preliminares la validaron antes de salir al aire, y su aplicación se dio según lo previsto.

Por su parte, respecto de "Sexo a lo Bien", no hay evidencias que permitan identificar respaldo científico que indicara rutas de acción, pero sí hubo estudios de línea de base para conocer la magnitud del problema y algunos estudios posteriores para saber qué había cambiado [33]; su punto de partida fue una problemática, algo de tipo fenoménico, distinta a la expresada por la comunidad más afectada; sus objetivos eran amplios y ambiciosos, pero no suficientemente medibles; su estrategia no obedeció a segmentos con criterios definidos ni mensajes centrados, pues si bien el foco en sexualidad responsable y el placer son positivos, más allá de ello hay efectos culturales que emergen en la vida sexual y de relación que sobrepasan el criterio del llamado a la responsabilidad y que, muchas veces, la adolescente no ve un problema en estar embarazada; al contrario, ellas sienten que su autoestima social se ve reforzada [34], dada la aceptación del fenómeno entre su grupo de referencia. Estas consideraciones cabe tomarlas en cuenta frente a los mensajes del llamado a la responsabilidad, como también se debe pensar en la amplitud del contenido en los mensajes, tratando a todos los grupos focalizados con cierta homogeneidad.

Otro aspecto es que, en dicha campaña, sus canales y mensajes fueron evaluados poco efectivos, lo que se le atribuye, en mercadeo, a la falta de segmentación. Tuvo imprevistos en su aplicación y funcionamiento, como el caso de la plataforma web, que presentó fallas de funcionamiento. A pesar de identificarse deficiencias a lo largo de todo el proceso, gran parte de las dificultades de "Sexo a lo Bien" se generaron a partir de la primera etapa y la segmentación, con consecuencias en las demás fases, lo que revela que la definición del problema, de los objetivos y de la estrategia misma, así como la especificidad en el público objetivo, tienen incidencia directa en los efectos de la campaña, y donde los marcos de referencia desempeñan un papel fundamental.

Si bien otros autores ya habían demostrado el éxito del mercadeo social en la promoción y la prevención de comportamientos saludables [6,22], con este estudio puede añadirse que los aspectos que permiten lograr efectos positivos son la claridad conceptual, la rigurosidad metódica y la coherencia con la que se formule y ejecute la campaña.

\section{Reconocimientos}

El equipo de trabajo agradece principalmente a la Universidad EAFIT por los aportes en especie (bases de datos) y tiempo de profesor investigador para la escritura de este artículo, con el apoyo de una estudiante de MBA. Igualmente, agradecemos a los revisores anónimos, quienes aportaron para mejorar el trabajo, así como nuestro reconocimiento al equipo editorial de la Revista por sus valiosos comentarios al manuscrito.

\section{Fuentes de financiación}

Se declara que para la realización de este trabajo no hubo fuentes de financiación.

\section{Conflictos de interés}

Los autores del presente artículo declaramos que no tenemos ninguna relación personal, científica, comercial o financiera con personas o instituciones, que pudiera sesgar los resultados presentados en el mismo.

\section{Referencias}

1 Organización Mundial de la Salud (oms). El embarazo en la adolescencia [internet]. 2014 sep. [citado 2015 abr. 17]. Disponible en: http://www.who.int/mediacentre/factsheets/fs364/es/

2 Profamilia. Encuesta Nacional de Demografía y Salud (ENDS) Tomo I. Componente demográfico [internet]. Bogotá: Ministerio de Salud, Profamilia, 2015 [citado 2018 feb. 15]. Disponible en: http://profamilia.org.co/docs/ENDS\%20\%20TOMO\%20I.pdf

3 Molina B, Diana P, Posada Z, Isabel C. Perspectiva de algunos actores sobre los modelos de educación para la salud subyacentes en programas de salud sexual y reproductiva dirigidos a adolescentes, Medellín 2004-2007. Rev. Fac. Nac. Salud Pública. 2013;31(1):19-29.

4 NIHCM Foundation. Recommended adolescent health care utilization: How social marketing can help. [internet]. 2009 mar. [citado 2015 mar. 23]. Disponible en: https://www.nihcm.org/pdf/ NIHCM-SocialMarketing-FINAL.pdf

5 Munoz Y. El mercadeo social en Colombia. Medellín: Fondo Editorial Universidad EAFIT; 2001. 
6 Evans WD, Silber-Ashley O, Gard J. Social marketing as a strategy to reduce unintended adolescent pregnancy. The Open Communication Journal. 2007;(1):1-8.

7 Sandoval JD, Rodríguez M., García GI, Gallo NE. Salud sexual y reproductiva en adolescentes de Medellín, 2006. Rev Salud Pública de Medellín. 2008;3(1):7-25.

8 Gaviria SL, Chaskel R. Embarazo en adolescentes de América Latina y el Caribe: impacto psicosocial. [internet] CCAP. s. a.;12(3):5-16 [citado 2018 mar. 4]. Disponible en: http://www. scp.com.co/precop-old/precop_files/ano12/TERCERO/embarazo_adolescentes.pdf

9 Gallo NE. Línea de base del proyecto de salud sexual y reproductiva para adolescentes en Medellín: componente cualitativo. Rev. Fac. Nac. Salud Pública 2009;27(3):282-90.

10 Flórez CE, Soto VE. Serie de estudios a profundidad ENDS 19902010. Factores protectores y de riesgo del embarazo adolescente en Colombia. [internet]. 2013 mar. [citado 2015 may. 4]. Disponible en: http://www.codajic.org/sites/www.codajic.org/files/Estudio $\% 20 \mathrm{a} \% 20$ pronfundidad $\% 20-\% 20$ Embarazo $\% 20$ adolescente $\% 20-$-pdf

11 Fletcher A, Harden A, Brunton G, Oakley O, Bonell C. Interventions addressing the social determinants of teenage pregnancy. Health Education. 2008;108(1):29-39.

12 Kegler M, Rodine S, LaDonna M, Oman RF, McLeroy K. An asset-based youth development model for preventing teen pregnancy: Illustrations from the HEART of OKC project. Health Education. 2003;103(3):131-44.

13 Lehr ST, Dilorio C, Dudley WN, Lipana JA. The relationship between parent-adolescent communication and safer sex behaviors in college students. J Fam Nurs. 2000;6(2):180-97.

14 DuRant R, Wolfson M, LaFrance B, Balkrishnan R, Altman D. An evaluation of a mass media campaign to encourage parents of adolescents to talk to their children about sex. J Adolesc Health. 2006;38(3):298-309.

15 Rodgers K. Parenting processes related to sexual risk-taking behaviors of adolescent males and females. J. Marriage Fam. 1999;61(1):99-109.

16 Unger JB, Molina GB, Teran L. Perceived consequences of teenage childbearing among adolescent girls in an urban sample. $\mathrm{J}$ Adolesc Health. 2000;26(3):205-12.

17 Sandfort T, Orr M, Hirsch J, Santelli J. Long-term health correlates of timing of sexual debut: Results from a National US Study. Am J Public Health. 2008;98(1):155-61.

18 Kim C, Rector R. Abstinence education: Assessing the evidence. Washington: The Heritage Foundation; 2008.

19 Weed S, Eriksen I, Lewis A, Grant G, Wibberly K. An abstinence program's impact on cognitive mediators and sexual initiation. Am J Health Behav 2008;32(1):60-73.

20 Slovic P, Finucane M, Peters E, MacGregor D. Risk as analysis and risk as feelings: Some thoughts about affect, reason, risk, and rationality. Risk Anal. 2004;24(2):311-22.
21 Witte K. Putting the fear back into fear appeals: The extended parallel process model. Commun. Monogr. 1992;59(4):329-349.

22 Colomer-Revuelta J, Grupo PredInfad/PAPPS Infancia y Adolescencia. Prevención del embarazo en la adolescencia. Rev Pediatr Aten Primaria. 2013;15(59):261-69.

23 Gómez-Dávila J, Castro-Mantilla M, Camacho-Hübner A. Sistematización del Proyecto Sol y Luna. Revista Salud Pública de Medellín. 2010;5(1):10-138.

24 Secretaría de Salud de Medellín - ASCODEs. Evaluación del impacto del programa de Salud Sexual y Reproductiva en Adolescentes de Medellín. Medellín: Alcaldía de Medellín; 2008.

25 Duque M, López Y. Prevención del embarazo adolescente en Medellín: ¿publicidad o comunicación? Folios 2009;18(20):105-13.

26 Davis KC, Blitstein J, Evans WD, Kamyab, K. Impact of a parentchild sexual communication campaign: Results from a controlled efficacy trial of parents. Reprodu Health. 2010;7(1):17.

27 Davis KC, Evans WD, Kamyab K. Effectiveness of a national media campaign to promote parent-child communication about sex. Health Educ. Behav. 2012;40(1):97-106.

28 Evans WD, Davis KC, Ashley OS \& Khan M. Effects of media messages on parent-child sexual communication. J Health Commun. 2012;17(5):498-514.

29 Fondo de Población de las Naciones Unidas (FPNU), Organismo Andino de Salud - Convenio Hipólito Unanue, Family Care International, Caedid. Buenas prácticas en prevención de embarazo adolescente. Colombia. [internet] s. d. [citado 2018 feb. 25]. Disponible en: http://www.planandino.org/bancoBP/sites/default/ files/Colombia\%20-Informe\%20BP\%20editado\%202010_0.pdf

30 Whitelaw S, Smart E, Kopela J, Gibson T, King V. Developing social marketing capacity to adress health issues. Health Education. 2011;111(4):319-31.

31 Munoz Y, Chebat JC \& Suissa JA. Using fear appeals in warning labels to promote responsible gambling among VLT Players: The key role of depth of information processing. J Gambl Stud. 2010;26(4):593-609.

32 Jaramillo-Palacio J. Políticas públicas de prevención del embarazo precoz en el marco de la realización de los objetivos de desarrollo del milenio: el caso de Medellín (Colombia) [Tesis Doctoral en Relaciones Internacionales Iberoamericanas] [Madrid]: Universidad Rey Juan Carlos; [internet] 2017 [citado 2018 feb. 23]. Disponible en: http://hdl.handle.net/10115/14849

33 Gallo NE, Molina AN. Evaluación de resultados inmediatos del proyecto Acciones de información, educación y comunicación en salud sexual y reproductiva para Medellín en 2007. Rev Salud Pública de Medellín. 2008;3(1):47-62.

34 Gómez-Sotelo Á, Gutiérrez-Malaver ME, Izzedin-Bouquet R, Sánchez-Martínez L, Herrera-Medina NE, Ballesteros-Cabrera M. Representaciones sociales de embarazo y la maternidad en adolescentes primigestantes y multigestantes en Bogota. Rev Salud Pública. 2012;14(2):189-99. 